\title{
The Natural Compound Dehydrocrenatidine Attenuates Nicotine-Induced Stemness and Epithelial-Mesenchymal Transition in Hepatocellular Carcinoma by Regulating a7nAChR-Jak2 Signaling Pathways
}

\author{
Ching-Li Li $\mathbb{D},{ }^{1,2}$ Chien-Che Wang $\mathbb{D}^{2},{ }^{2}$ Hsin-Te Pai $\mathbb{D},{ }^{2}$ Stan-Ley Tu $\mathbb{D}^{2},{ }^{2}$ Pin-Yuan Hou $\mathbb{D}^{2},{ }^{2}$ \\ Chien-Yu Huang, ${ }^{3,4,5,6}$ Ming-Te Huang $\mathbb{D}^{1,7}$ and Yu-Jia Chang $\mathbb{D}$ 1,5,8,9 \\ ${ }^{1}$ Graduate Institute of Clinical Medicine, College of Medicine, Taipei Medical University, Taipei 110, Taiwan \\ ${ }^{2}$ Department of Surgery, Pojen General Hospital, Taipei City 105, Taiwan \\ ${ }^{3}$ Department of Surgery, Taipei Medical University, Shuang Ho Hospital, New Taipei City 235, Taiwan \\ ${ }^{4}$ Department of Surgery, School of Medicine, College of Medicine, Taipei Medical University, Taipei 110, Taiwan \\ ${ }^{5}$ Department of Pathology, Wan Fang Hospital, Taipei Medical University, Taipei 116, Taiwan \\ ${ }^{6}$ Division of Colorectal Surgery, Department of Surgery, Taipei Medical University Shuang Ho Hospital, \\ New Taipei City 235041, Taiwan \\ ${ }^{7}$ Department of Surgery, Xin Tai General Hospital, New Taipei City 235, Taiwan \\ ${ }^{8}$ Cancer Research Center and Translational Laboratory, Department of Medical Research, Taipei Medical University Hospital, \\ Taipei Medical University, Taipei 110, Taiwan \\ ${ }^{9}$ Cell Physiology and Molecular Image Research Center, Wan Fang Hospital, Taipei Medical University, Taipei 116, Taiwan
}

Correspondence should be addressed to Ming-Te Huang; mthuant@tmu.edu.tw and Yu-Jia Chang; r5424012@tmu.edu.tw

Received 31 October 2021; Accepted 16 December 2021; Published 24 January 2022

Academic Editor: Drenka Trivanović

Copyright (c) 2022 Ching-Li Li et al. This is an open access article distributed under the Creative Commons Attribution License, which permits unrestricted use, distribution, and reproduction in any medium, provided the original work is properly cited.

Background. Exposure to nicotine has been observed associated with tumor progression, metastasis, and therapy resistance of many cancers. Hepatocellular carcinoma (HCC) is one major cancer related to the liver and the most difficult to treat malignancies worldwide. The underlying mechanism of nicotine in the stimulation of HCC tumorigenesis is still not studied well. Methods. Classically, nicotine binds to nicotinic acetylcholine receptors (nAChRs) and induces many downstream cancerassociated signaling pathways. Big data analysis is used to explore the importance of a7nAChR-Jak2 axis in the progression of hepatocellular carcinoma. Bioinformatic analysis was performed to determine gene associated with a7nAChR-Jak2 axis of HCC patients. Biological importance of a7nAChR-Jak2 axis was investigated in vitro (Hun7 and HepG2 cell lines), and athymic nude mouse models bearing HepG2-HCC cells xenografts were established in vivo. Result. We found that nicotine exposure stimulated the HCC tumorigenicity by inducing the expression of one of the key nAChRs subunit that is $\alpha 7 \mathrm{nAChR}$ as well as the expression of Janus kinase (JAK)-2. In both the in vitro and in vivo studies, the reduced overexpression of $\alpha 7 \mathrm{nAChR}$ and increased sensitization of HCC towards treatment is observed with dehydrocrenatidine (DHCT), a novel and potent JAK family kinase inhibitor. Interestingly, DHCT treatment results in the reduction of the epithelial-mesenchymal transition process which leads to a significant reduction of clonogenicity, migratory, and invasive ability of HCC cells. Moreover, DHCT treatment also inhibits the cancer stem cell phenotype by inhibiting the tumor-sphere formation and reducing the number of ALDH1+ cells population in nicotine-stimulated HCC cells. Conclusions. Taken together, the presented results indicate the positive effect of inhibition of nicotine induced overexpression of $\alpha 7 \mathrm{nAChR}$ and JAK2, unique to HCC. Thus, these findings suggest the nicotine effect on HCC progression via $\alpha 7 \mathrm{nAChR}$-mediated JAK2 signaling pathways, and DHCT treatment enhances the therapeutic potential of HCC patients via overcoming/reversing the effect of nicotine in HCC patients. 


\section{Introduction}

Currently, over a billion people in the world, including approximately $19 \%$ of adults, actively smoke tobacco [1]. Smoking releases more than 4000 harmful chemicals, which adversely affects almost all organs [2], including the liver [2]. Smoking is a well-known risk factor for many diseases, including cancers [3]. However, how cigarette smoking contributes to the development of liver cancer remains underinvestigated.

Nicotine, which is a major addictive chemical component of cigarette smoking, poses much serious health; this compound influences numerous biological function such as modulation of gene expression, DNA damage, oxidative stress, proliferation, apoptosis, angiogenesis, and regulation of secretion of hormones [4]. This compound exerts its effects via stimulation of the nicotinic acetylcholine receptors (nAChRs) that are ligand-activated ion channels [5]. Among various subtypes of $\mathrm{nAChR}$ receptors, alpha7subtype of nAChR ( $\alpha 7 \mathrm{nAChR}$ ) has been seen to exert special significance on the cellular process $[6,7] . \alpha 7 \mathrm{nAChR}$ is expressed and it is involved in regulation of a variety of human cancer and normal cells and tissues, such as liver cancer $[6,7]$. It modulates many cancer-associated properties in most cancers and interestingly plays a vital role in the regulation of inflammatory markers expression, which regulates various other pathological conditions [8-11]. Such a broad role of $\alpha 7 \mathrm{nAChR}$ and the effect of nicotine have a significant impact in defining the long-term faith of different cancers.

It is noticed that the number of different nicotine-related malignancies is on the rise [12]. Among, them, hepatocellular carcinoma (HCC) is one of the most common, and often diagnosed malignancies, which ranks number the second leading reason of cancer-related mortality worldwide with approximately 800,000 new cases reported every year [13]. Despite advancements in early detection and diagnostic techniques and treatment modalities, the prognosis of HCC patients remained poor even after the curative liver resection [14]. Several epidemiologic studies have reported that smoking tobacco is connected with HCC development [15]. However, the biological effect of tobacco and the underlying molecular mechanism in HCC remain elusive.

In the previous study by our group, we demonstrated by applying the HCC patient's clinical samples that smoking is an independent risk factor for HCC progression through the $\alpha 7 \mathrm{nAChR}$ and JAK2 signalling [16]. In the cholinergic system, $\alpha 7 \mathrm{nAChRs}$ facilitates many different pathways, it is extensively studied in association with the antiinflammatory pathways [17]. In monocytes and macrophages, $\alpha 7 \mathrm{nAChR}$ triggers and attracts the cytosolic JAK2 protein, leading to the initiation of the formation of a heterodimer complex that triggers STAT3 signal transduction [18]. The JAK2/STAT3 signaling pathways also play a critical role in the growth, proliferation, and metastasis of cancers [19]. The JAK2-modulated STAT3 transcription factors are involved in many processes, such as immune response, inflammation, and cancer progression, by activating proinflammatory cytokines, growth factors, and onco- genes $[20,21]$. Apart from $\alpha 7 \mathrm{nAChR}$ negative regulatory role in the inflammatory response, the role of $\alpha 7 \mathrm{nAChR}$ together with JAK2, in connection with exposure to nicotine in HCC, remains unclear. This prompted us to identify new potential drugs to overcome the effects of nicotine on HCC. For example, the natural compound dehydrocrenatidine (DHCT), a novel and potent JAK family kinase inhibitor that modulates JAK/STAT3 expression [22] in HCC, can sensitize HCC cells against nicotine.

In the current study, we investigated the functional significance of nicotine and $\alpha 7 \mathrm{nAChR}$ in HCC progression and therapy resistance, the molecular mechanisms underlying its oncogenic role in HCC, and the potential of DHCT treatment to reverse the effects of nicotine in HCC.

\section{Materials and Methods}

2.1. Chemicals and Reagents. Dehydrocrenatidine, $\geq 98 \%$ purity, was purchased from AOBIOUS (CAS No: 6523662-6, AOBIOUS), dissolved in dimethyl sulfoxide (DMSO) to $20 \mathrm{mg} / \mathrm{mL}$ as stock solution and kept at $-20^{\circ} \mathrm{C}$ until use. Nicotine, $\geq 99 \%$ (GC), liquid, purity was purchased from Sigma-Aldrich (CAS No: 54-11-5).

2.2. Cell Lines and Culture Media. The human Huh7 and HepG2 HCC cell lines were purchased from the American Tissue Culture Collection and cultured in Roswell Park Memorial Institute (RPMI) 1640 Medium (Thermo Fisher Scientific, Waltham, MA, USA) supplemented with $10 \%$ fetal bovine serum (FBS) and 1\% penicillin-streptomycin (Invitrogen, Life Technologies) at $37^{\circ} \mathrm{C}$ in a $5 \%$ humidified $\mathrm{CO}_{2}$ incubator.

2.3. Microarray and RNAseq Preprocessing and Analysis. Gene expression profiles, GSE14323, were downloaded from the Gene Expression Omnibus database (http://www.ncbi. nlm.nih.gov/geo/), and expression of $\alpha 7 \mathrm{nAChR}$ and JAK2 was analyzed using the Oncomine platform (https://www. oncomine.org/resource/) [23].

2.4. Cell Viability Test. The DHCT stock was prepared by dissolving $20 \mathrm{mg} / \mathrm{mL}$ of the mixture in DMSO. The stocks of each drug were stored at $-20^{\circ} \mathrm{C}$ until use. The effects of DHCT on cell proliferation were detected using the Cell Counting Kit-8 (CCK-8) assay (Dojindo Laboratories, Kumamoto, Japan), according to manufacturer's instructions.

2.5. Cell Cycle Analysis. The distribution of cell cycles was determined by staining cells with the propidium iodide (PI), after treatment, according to manufacturer's instructions. To determine the effect on the cell cycle, HCC cells were exposed to DHCT for $48 \mathrm{~h}$. Next, the cells were washed, fixed with $70 \%$ ethanol, washed again, resuspended, and stained with $10 \mu \mathrm{g} / \mathrm{mL}$ of PI in PBS for $30 \mathrm{~min}$ at room temperature in the dark. The cells were analyzed through by flow cytometry (Becton Dickinson, Mountain View, CA, USA), and the population of cells in each phase was counted.

2.6. Aldehyde Dehydrogenase Assay. Aldehyde dehydrogenase (ALDH) activity in HCC cells was assayed using the 
ALDEFLUOR kit according to manufacturer's instructions (STEMCELL Technologies, Durham, NC, USA). The cells that showed positive activity for ALDH1 were isolated and analyzed. Briefly, the human HCC cell lines Huh7 and HepG2 were suspended at $1 \times 106$ cells/mL in ALDEFLUOR assay buffer containing the ALDH substrate (BAAA, $1 \mu \mathrm{mol} /$ $\mathrm{L}$ per $1 \times 106$ cells) and incubated for $40 \mathrm{~min}$ at $37^{\circ} \mathrm{C}$. Cells incubated with the ALDEFLUOR substrate and treated with $50 \mathrm{mmol} / \mathrm{L}$ of diethylaminobenzaldehyde (DEAB), a specific ALDH inhibitor, were used as a reference control. Cells stained with PI alone were used as negative controls, and ALDEFLUOR-stained cells treated with DEAB and those stained with the secondary antibody alone were considered viable.

\subsection{Western Blotting and Quantitative Reverse-Transcription} Polymerase Chain Reaction. After the corresponding treatment, the HCC (Huh7 and HepG2) cells were washed with PBS and then lysed in RIPA lysis buffer. Cellular protein lysates were isolated using a protein extraction kit (QIAGEN, USA) and quantified using the Bradford protein assay kit (Beyotime, Beijing, China). Approximately $20 \mu \mathrm{g}$ of sample from different experiments was loaded and subjected to SDS-PAGE by using the Mini-PROTEAN III system (BioRad, Taiwan). Separated proteins were transferred onto a polyvinylidene fluoride (PVDF) membrane by using the Trans-Blot Turbo Transfer System (Bio-Rad), which was followed by blocking with Tris-buffered saline plus skimmed milk. These PVDF membranes were then probed with the respective primary antibodies followed by the secondary antibody. An enhanced chemiluminescence detection kit was used to detect the proteins of interest. Images were captured and analyzed using the UVP BioDoc-It system (Upland, CA, USA). Quantitative reverse-transcription (RT) polymerase chain reaction (PCR) was performed by isolating total RNA using TRIzol-based protocol (Life Technologies) provided by the manufacturer. In brief, $1 \mu \mathrm{g}$ of total RNA was reverse transcribed using a QIAGEN OneStep RT-PCR kit (QIAGEN, Taiwan), and PCR was performed using a Rotor-Gene SYBR Green PCR kit (400; QIAGEN, Taiwan). The Commercial antibodies and primers used in this study are shown in Supplementary Table S1 and Supplementary Table S2, respectively.

2.8. Colony Formation Assay. As per the protocol for colony formation assay described previously [24], 400 liver cancer cells were seeded in six-well plates and treated with DHCT. The cells were allowed to grow for another week and then harvested, fixed, and counted. The image was captured and quantified using ImageJ software (https://imagej.nih.gov/ij/ download.html).

2.9. Wound Healing Migration Assay. The cells were seeded in six-well plates (Corning, Corning, NY, USA) with RPMI 1640 medium containing 10\% FBS after the cells reached 95\%-100\% confluence. A scratch along the median axis was then made with a sterile yellow pipette tip across cells. Cell migration pictures of cells with or without DHCT treatment were captured at 0 and $48 \mathrm{~h}$ under a microscope and analyzed using NIH Image J software (https://imagej.nih. gov/ij/download.html).

2.10. Matrigel Invasion Assay. Cells $\left(3 \times 10^{5}\right)$ were seeded in 24 -Transwell chambers with an $8 \mu \mathrm{m}$ pore membrane coated with Matrigel in the upper chamber of the Transwell system containing serum-free RPMI 1640 medium. The lower chamber of the Transwell system contained the medium with $20 \%$ FBS. After incubation at $37^{\circ} \mathrm{C}$ for $6 \mathrm{~h}$, noninvaded HCC cells on the upper side of the membrane were carefully removed with a cotton swab, and the invaded cells were stained with crystal violet dye, air-dried, and photographed under a microscope. Images were analyzed using NIH Image J software (https://imagej.nih.gov/ij/download.html).

2.11. Sphere Formation Assay. Cells $\left(5 \times 10^{3} /\right.$ well $)$ were plated in ultralow-attachment six-well plates (corning) containing stem cell medium consisting of serum-free RPMI 1640 medium supplemented with $10 \mathrm{ng} / \mathrm{mL}$ of human basic fibroblast growth factor (bFGF; Invitrogen, Grand Island, NY, USA), $1 \times$ B27 supplement, and $20 \mathrm{ng} / \mathrm{mL}$ epidermal growth factor (Invitrogen). The medium was changed every $72 \mathrm{~h}$. After 14 days of incubation, the formed spheres were counted and photographed.

2.12. Animal Studies. This study was approved by the Institutional Animal Care and Use Committee (IACUC) of Taipei Medical University. All procedures were performed according to guidelines of IACUC, and all efforts were made to minimize animal suffering and the number of animals used. Twenty-seven male athymic nude mice at 4 weeks of age were used for this study. The mice were divided into three groups ( $N=6$ per group) and repeat experiments 3 times. The mice were maintained under pathogen-free conditions and were provided with sterilized food and water. The antiproliferative effect of DHCT on HepG2HCC cells in vivo was investigated by using HepG2 cell xenograft mouse model of human HCC carcinoma. First, $1 \times 10^{6} \mathrm{HepG}_{2}$ cells along with proper controls were subcutaneously injected into the right flank near the hind leg of each nude mouse. When the tumor was palpable (tumor volume of approximately $100 \mathrm{~mm}^{3}$ ), they were randomly divided into control $(100 \mu \mathrm{L}$ of normal saline [NS] by intraperitoneal injection plus $100 \mu \mathrm{L}$ of $1 \%$ DMSO), nicotine was administered, and the role of DHCT treatment in overcoming the effect of nicotine $(200 \mathrm{mg} / \mathrm{kg} /$ day by intraperitoneal injection plus $100 \mu \mathrm{L}$ of $1 \%$ DMSO and $0.5 \%$ CMC-Na sterile water). The treatments were performed 5 times/week for 4 weeks. Tumor volume was measured using a standard vernier calliper, the tumor volume was calculated using the established formula. Tumor volume $=1 / 2$ (length $\times$ width2) in $\mathrm{mm}^{3}$ [25]. Upon the completion of the experiments, all the mice were euthanized by $\mathrm{CO}_{2}$ asphyxiation followed by cervical dislocation, and the tumors were then immediately removed, weighted ex vivo and photographed for further analysis.

2.13. Immunohistochemistry (IHC) Staining. The tumor tissue was fixed in 10\% neutral-buffered formalin, embedded in paraffin wax, and cut into sections of $3 \mu \mathrm{m}$ thickness. 


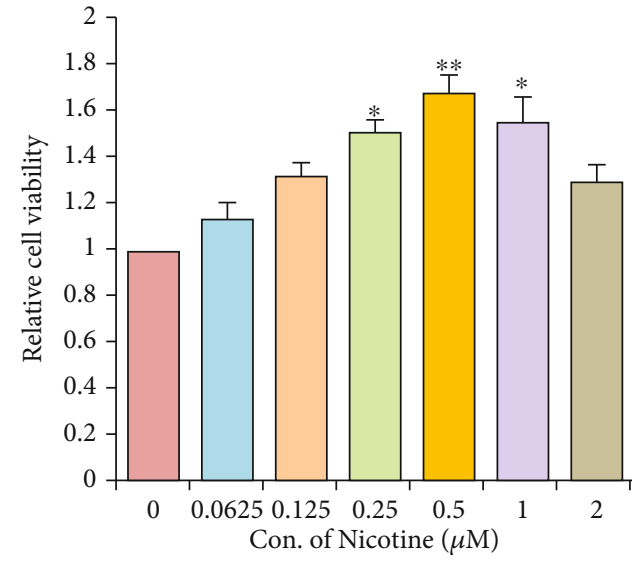

(a)

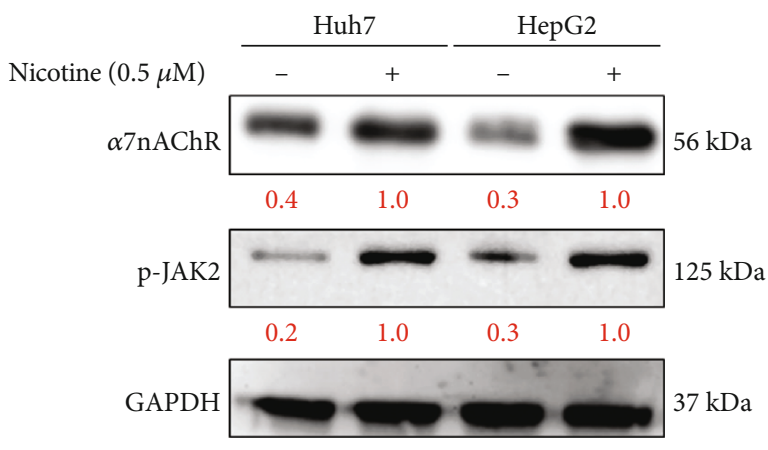

(c)

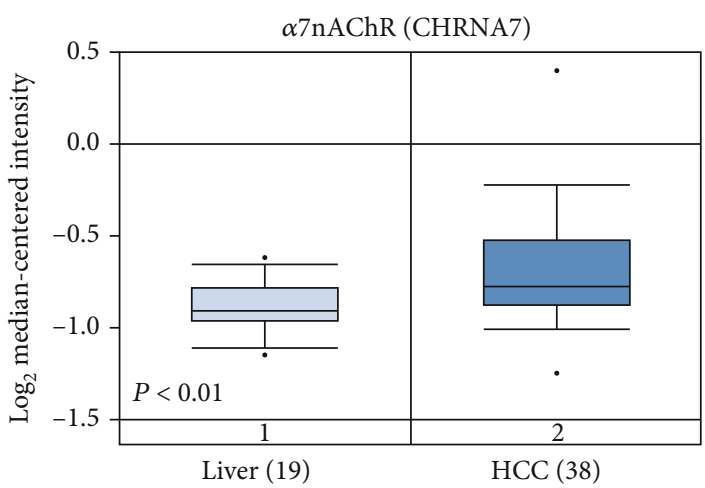

(e)

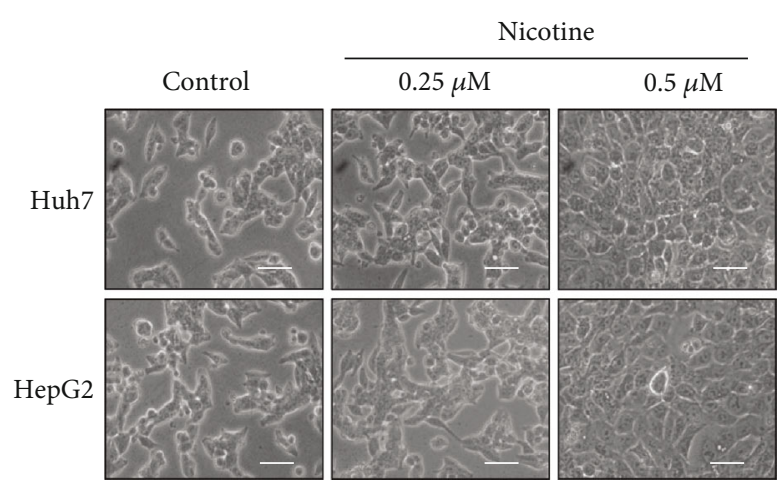

(b)

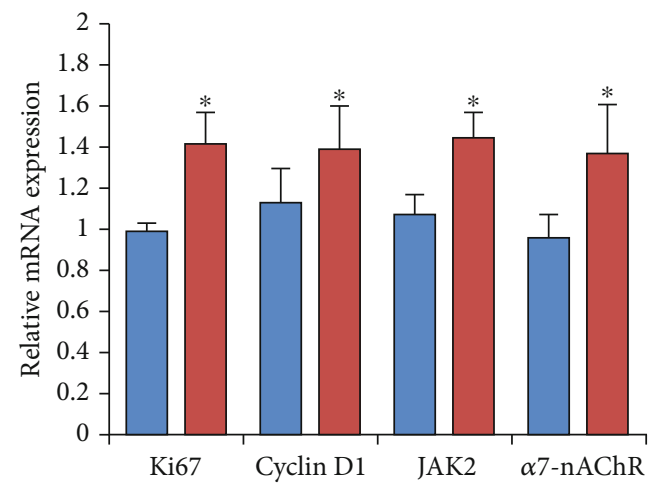

$\square$ CTL

$\square$ Nicotine

(d)

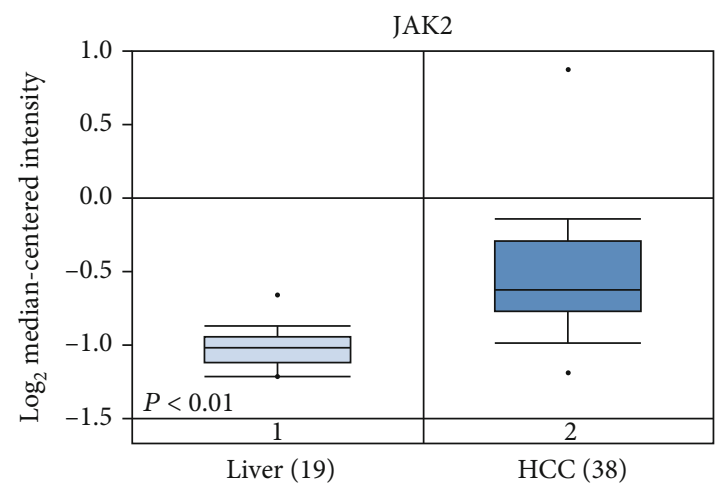

(f)

Figure 1: Continued. 


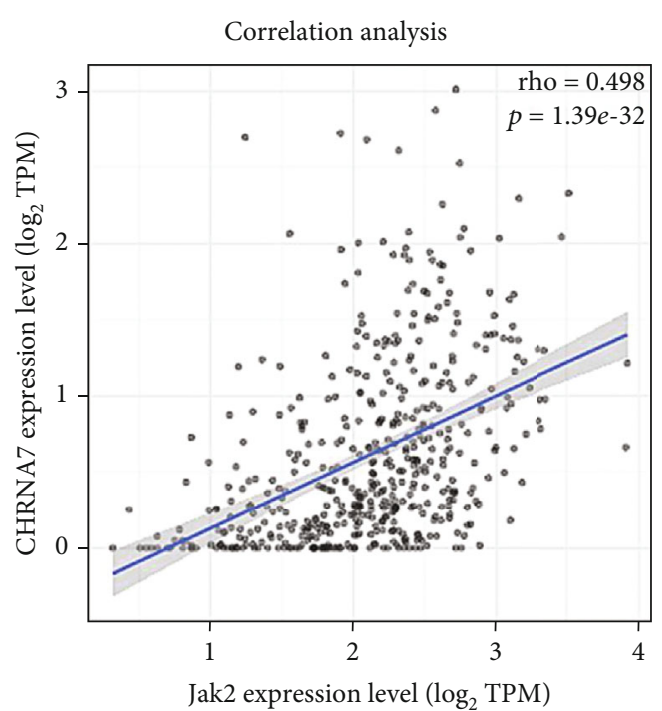

(g)

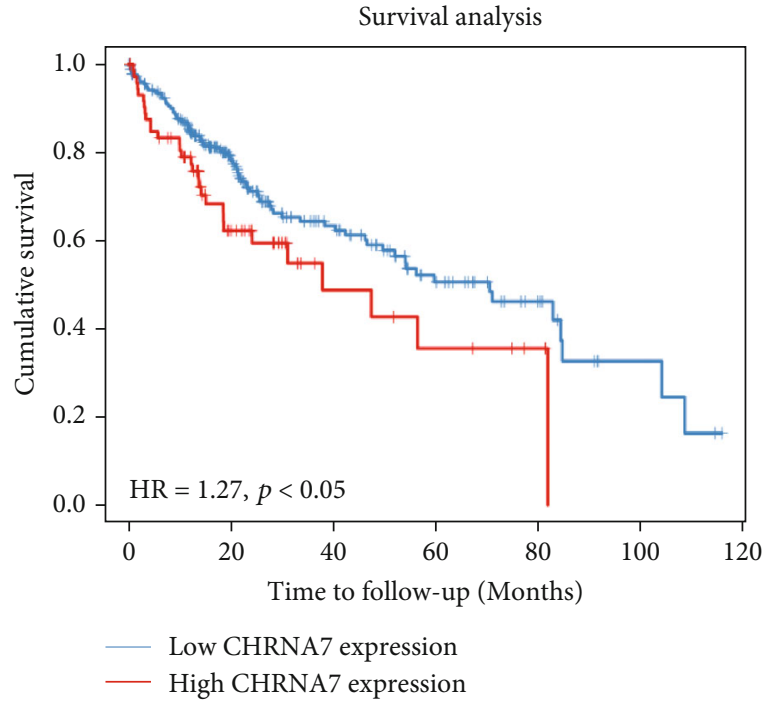

(h)

Figure 1: Nicotine treatment promotes cell proliferation of HCC cells. (a) Huh7 and HepG2 cells $\left(5 \times 10^{4}\right.$ cells $)$ were treated with the indicated concentrations of nicotine for $48 \mathrm{~h}$. The cell viability of Huh7 and HepG2 was determined by the CCK-8 assay. (b) Optical microscopy showed nicotine treatment at $0.25-0.5 \mu \mathrm{M}$ dose-dependently induced morphological changes of HCC (Huh7 and HepG2) cells. Scale bar $100 \mu \mathrm{m}$. (c) Huh7 and HepG2 cells stimulated with $0.5 \mu \mathrm{M}$ nicotine for $48 \mathrm{~h}$ were harvested, and the effects of nicotine on $\alpha 7 \mathrm{nAChR}$ and JAK2 expression were determined by western blot analysis. (d) Real-time PCR analysis of Ki67, Cyclin D1, JAK2, and $\alpha 7 \mathrm{nAChR}$ mRNA in HCC cells. (e, f) Expression of $\alpha 7 \mathrm{nAChR}$ (CHRNA7) and JAK2 in liver cancer (GSE14323). (g) Correlation analysis of $\alpha 7 \mathrm{nAChR}$ (CHRNA7) expression with the JAK2 expression in LIHC TCGA patient data. (h) Kaplan-Meier analysis of expression of $\alpha 7 \mathrm{nAChR}$ (CHRNA7) and JAK2 for LIHC patients based on the result from the public TCGA data. GAPDH was used as an internal control. Bar represent the mean $\pm \mathrm{SD}$ values of three independent experiments; ${ }^{*} p<0.05 ;{ }^{* *} p<0.01$.

Then, the sections were immunohistochemically stained with anti-Ki67 and anti-cleaved (cl)-Caspase-3 antibodies (Cell Signaling Technology) at $4^{\circ} \mathrm{C}$ overnight. Slides were rinsed with phosphate-buffered saline (PBS) and incubated at room temperature for 30 minutes with biotinylated goat anti-mouse IgG secondary antibody (Dako, Glostrup, Denmark). After washing in Tris-hydrochloride acid buffers (TBS), the slides were incubated with streptavidinperoxidase reagent (Dako) and treated with $3,3^{\prime}$-diaminobenzidine (DAB; Sigma Aldrich; Merck $\mathrm{KGaA}$ ) for 5 minutes. Finally, sections were rinsed with $\mathrm{ddH} 2 \mathrm{O}$ and counterstained with hematoxylin. Slides were observed under microscope, with the selection of 5 fields of view randomly. The final percentage of positive cells was calculated with the Motic Image software (version 1.2; Micro-Optical Group Co.)

2.14. Terminal Deoxynucleotidyl Transferase dUTP Nick-End Labeling (TUNEL) Assay. Apoptotic cell death in the HCC tumor tissue was determined by TUNEL assay using an Apoptag ${ }^{\circledR}$ Peroxidase In Situ Apoptotic Detection Kit (Roche according to manufacturer's protocol). Quantification was performed by calculating the percentage of TUNEL-positive cells by using a fluorescence microscope. The results are expressed as the mean number of TUNELpositive apoptotic HCC cells in each group.
2.15. Statistical Analysis. All assays were performed at least three times in triplicate. Values are expressed as mean \pm standard deviation (SD). Comparisons between groups were estimated using Student's $t$-test for cell line experiments or the Mann-Whitney $U$-test for clinical data. The KaplanMeier method was used for survival analysis, and the difference between survival curves was tested with a log-rank test. All statistical analyses were performed using IBM SPSS Statistics for Windows, version 20 (IBM, Armonk, NY, USA). $p<0.05$ was considered statistically significant.

\section{Results}

3.1. Nicotine Augments Cell Proliferation Abilities of Huh7 and HepG2 Human HCC Cells through $\alpha 7 n A C h R-J A K 2$. First, we examined the effect of nicotine on HCC cell proliferation, both the Huh7 and HepG2 cells were titrated with the 0.0625 to $2 \mu \mathrm{M}$ concentration of nicotine for $48 \mathrm{~h}$, and cell viability of these cells was determined by CCK- 8 assay. The result showed that, as compared to control (PBS treated), nicotine treatment augments the human HCC (Huh7 and HepG2) cell proliferation (Figures 1(a) and 1(b)). In vitro, nicotine stimulates the proliferation of HCC cells through $\alpha 7 \mathrm{nAChR}$. Meanwhile, Western blot results also indicated, as shown in Figure 1(c), nicotine treatment at $0.5 \mu \mathrm{M}$ and stimulated the expression of p-JAK2 and 
$\alpha 7 \mathrm{nAChR}$ in both the Huh7 and HepG2 cells. Real-Time Quantitative Reverse Transcription (qRT)-PCR analysis of the mRNA expression level showed the level of Ki67, Cyclin D1, JAK2, and $\alpha 7 \mathrm{nAChR}$ in Huh7 and HepG2 cells was significantly higher in the nicotine-treated cells compared to the control-treated group (Figure 1(d); ${ }^{*} p<0.01$ ). Furthermore, bioinformatics analysis of publicly available dataset (GSE14323) by using Oncomine an online tool (https:// www.oncomine.org/resource/) [23] also demonstrated (Figures 1(e) and 1(f)) the expression of JAK2 and $\alpha 7 \mathrm{nAChR}$, considerably higher in the HCC patients samples compared to the normal counterpart, whereas the expression of $\alpha 7 \mathrm{nAChR}$ correlates positively with the expression of JAK2 expression in HCC patients' samples (Figure 1(g)), and the poor overall survival of HCC patients was observed in patients exhibiting higher expression of $\alpha 7 \mathrm{nAChR}$ (Figure 1(h)). All of these in vitro and in silico results indicated the nicotine treatment induces the HCC tumorigenesis by inducing the expression of $\alpha 7 \mathrm{nAChR}$-JAK2.

\subsection{Nicotine Enhances Migratory, Invasive, Self-Renewal,} and EMT Processes of HCC Cells. Furthermore, we evaluated the effect to evaluate the effect of nicotine treatment on HCC cell migratory, invasive, self-renewal, metastasis, and other malignant biological behaviour. The result indicates nicotine $(0.5 \mu \mathrm{M})$-treated cells after $24 \mathrm{~h}$ stimulated the Huh7 and HepG2 cells to migrate more quickly and close the scratch wounds (Figure 2(a)). Furthermore, we conducted a Transwell invasion assay to evaluate the effect of nicotine on the invasive properties of HCC cells. The results demonstrated that both the HCC, Huh7, and HepG2 cells significantly enhanced the ability to invade through the Matrigel matrix, and the number of cells that invaded through the Matrigel matrix was significantly higher after the nicotine treatment, compared to the control (Figure 2(b)). Studies have shown that cancer stem cells (CSCs) are characterized by unlimited proliferative properties and showing uncontrolled cellular growth [23]. Here, we performed the colony-formation and tumor-sphere abilities of nicotine-treated HCC cell (Huh7 and HepG2) results and demonstrated that nicotine treatment significantly induced the colony and tumor-sphere generating abilities of treated HCC cells compared to the control group (Figures 2(c) and 2(d)). The epithelial to mesenchymal transition (EMT) process indicates the tumor invasiveness and angiogenesis of cancer cells. Hence, we investigated the expression of markers associated with the EMT process together with the expression status of p-JAK2 and cancer stem cell markers' (CSCs) markers. Western blot image depicted in Figure 2(e) results demonstrated that the expression level of epithelial-marker vimentin (Vim) and CSC's marker (CD133) was induced together with the expression of p-JAK2 after the nicotine exposure of both the HCC (Huh7 and HepG2) cells.

3.3. Dehydrocrenatidine (DHCT) Treatment Reversed the Nicotine Effect on Human HCC Cells via Targeting the Expression of $\alpha 7 n A C h R-J A K 2$. To examine the potential role of DHCT as a potential drug that targets $\alpha 7 \mathrm{nAChR-JAK2}$ may reverse the effect of nicotine from tobacco smoke in
HCC (Huh7 and HepG2) cells. DHCT, a novel and potent JAK family kinase inhibitor, inhibits JAK/STAT3 expression [26] (Chemical structure of DHCT, Figure 3(a)). Furthermore, we treated Huh7 and HepG2 cells with the serial dose of DHCT. As shown in Figure 3(b), the $\mathrm{IC}_{50}$ value of DHCT treatment on Huh7 $\left(\mathrm{IC}_{50}: 34.4 \mu \mathrm{M}\right)$ and HepG2 $\left(\mathrm{IC}_{50}\right.$ : $36.87 \mu \mathrm{M})$ cells sensitizes towards nicotine, to confirm the effect of DHCT effect on the expression of $\alpha 7 \mathrm{nAChR}$ and JAK2. The result of Western blot analysis (Figure 3(c)) demonstrated $\alpha 7 \mathrm{nAChR}$ and JAK2 expression was significantly inhibited in DHCT treated cells compared to the control (nicotine only treated cells). Interestingly, the mRNA expression levels of Ki67, Cyclin D1, JAK2, and $\alpha 7 \mathrm{nAChR}$ significantly inhibited on both the Huh7 and HepG2 HCCDHCT-treated cells as compared to control-treated (nicotine only) (Figure $3(\mathrm{~d}) ;{ }^{* *} p<0.01$ ). Furthermore, the cell cycle was analyzed as the percentage of cells at each stage of the cell cycle arrest after DNA staining with PI. The effect of DHCT $\left(\mathrm{IC}_{20}\right)$ on the cell cycle distribution was observed by FACS analysis, as described in Figure 3(e), after $48 \mathrm{~h}$ of DHCT exposure. The HCC cells showed a higher percentage of arrest at G0/G1 phase (Huh7: 47\%; HepG2: 44\%) and a lower number of cells at the G2/M phase (Huh7: 17\%; HepG2: 14\%) compared to the control nicotine only treated cells, the lower number of cells at G0/G1 phase (Huh7: 51\%; HepG2: $49 \%$ ), and the higher number of cells G2/M phase (Huh7: 10\%; HepG2: 8\%), indicating the DHCT important role in neutralizing the nicotine effect on HCC cells.

3.4. DHCT Treatment Inverses the Nicotine Effect on HCC Cell Migratory, Invasive, and EMT Properties. Additionally, we examined the effect of DHCT on oncogenic properties of nicotine-stimulated HCC cells. The effect of DHCT on the migratory and invasive properties of HCC cells (Huh7 and HepG2) was investigated. Treatment with DHCT $\left(\mathrm{IC}_{20}\right)$ for $48 \mathrm{~h}$ strongly reduced the migration (Figure $4(\mathrm{a})$ ) and invasion (Figure 4(b)) capacity of cells, showing that JAK/STAT3 inhibitor effectively reduced the motility and invasiveness of nicotine stimulated HCC cells when compared with the nontreated control counterpart. EMT plays a key role in the invasion and metastasis of HCC cells [27]. Furthermore, the effect of DHCT on EMT properties and expression of EMT markers, E-cadherin and vimentin, were determined through Western blotting (Figure 4(c)). The results indicated the increased expression of E-cadherin and reduced expression of vimentin. These results advocated the DHCT effects in preventing and neutralizing the effect of nicotine on the migratory and invasive potential of HCC cells.

3.5. DHCT Treatment Modulates the Nicotine Effect on Tumorigenicity and Self-Renewal Abilities of HCC Cells. To further examine the effect of DHCT in modulating the effect of nicotine on tumorigenic properties of HCC cells, we assayed the colony and tumor sphere formation of the human HCC cell lines Huh7 and HepG2. Colony and tumor sphere formation assays are important for the identification of stemness $[28,29]$. DHCT treatment suppressed tumor sphere and colony formation of HCC cells (Figures 5(a) 

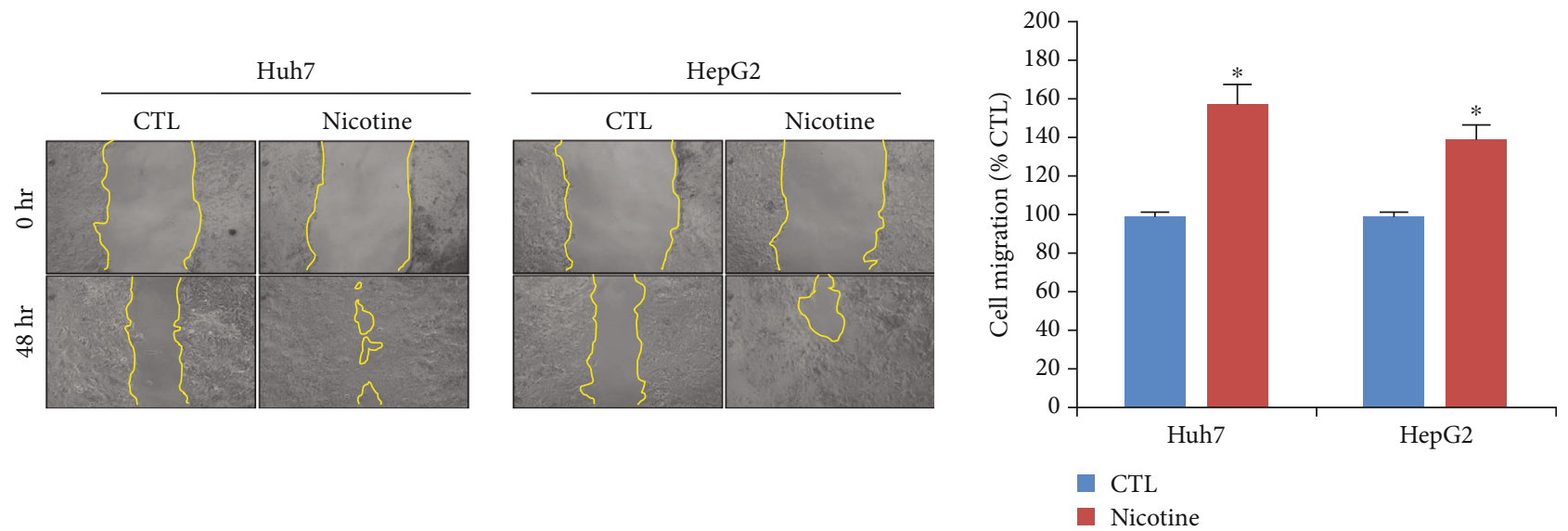

(a)
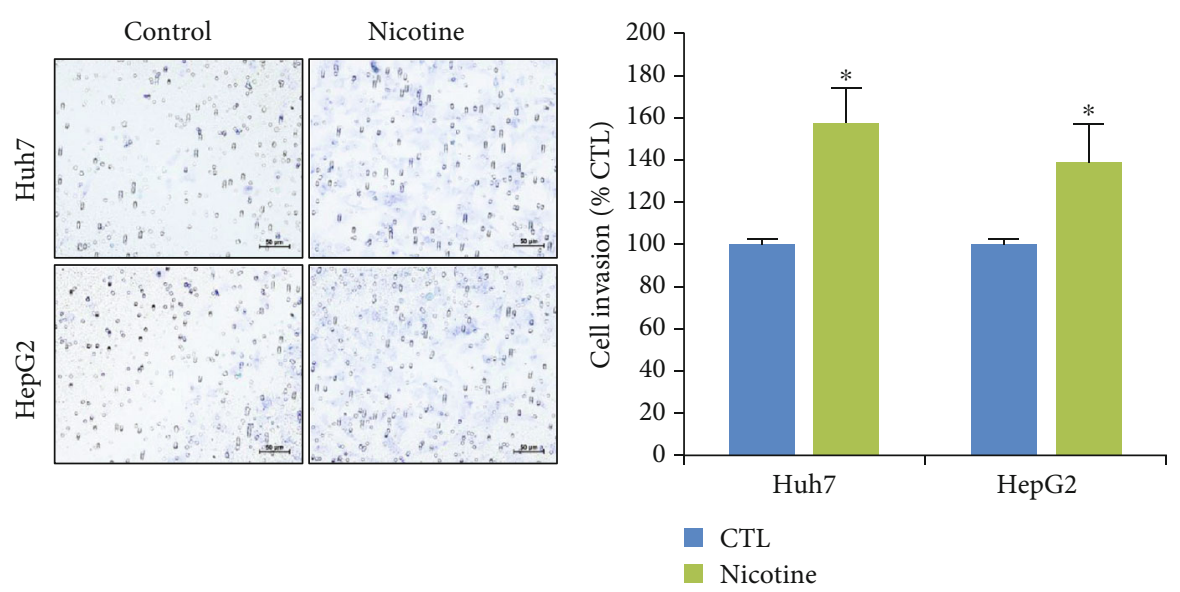

(b)
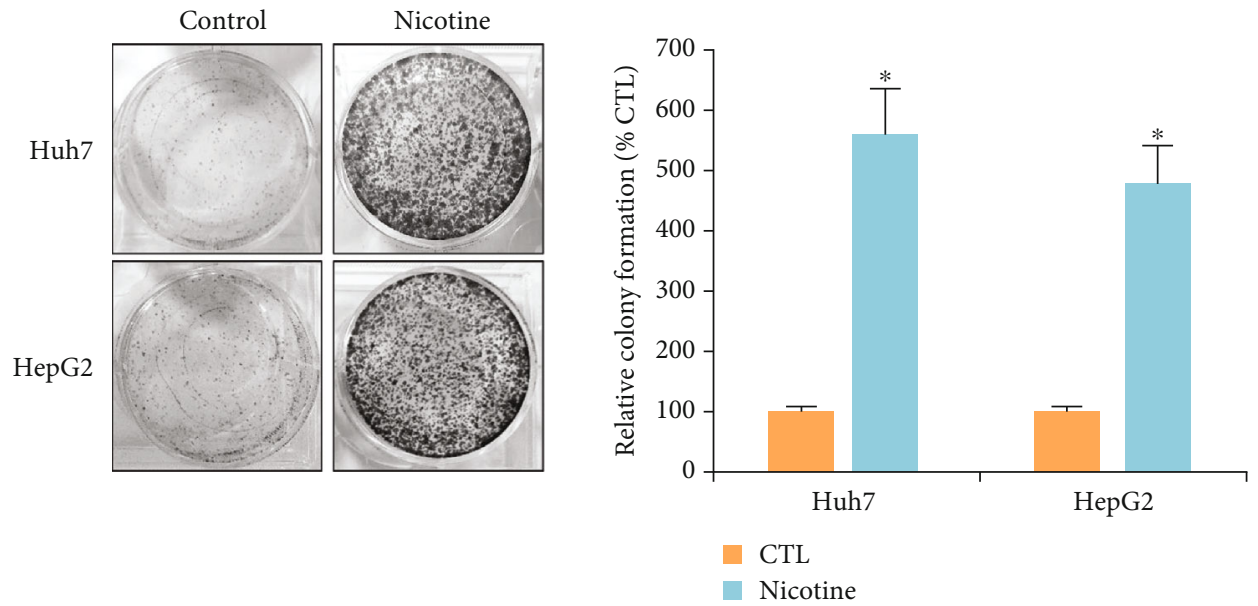

(c)

Figure 2: Continued. 


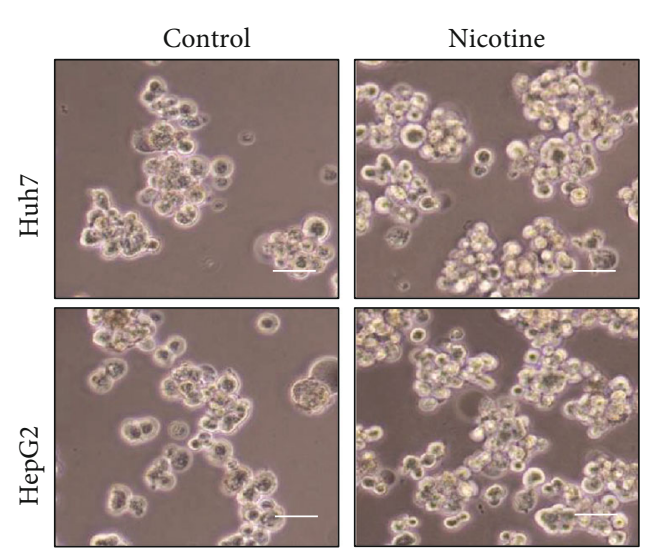

(d)

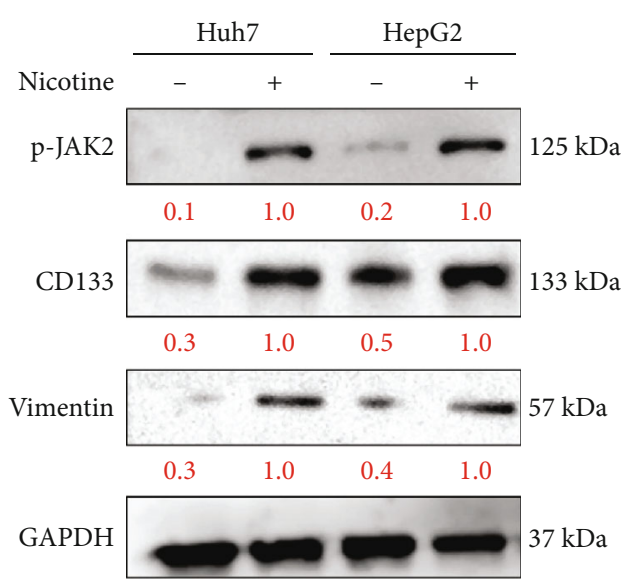

(e)

Figure 2: Nicotine induces Huh7 and HepG2 HCC cell migratory, invasive, self-renewal, and EMT properties in vitro. (a, b) Wound healing and Transwell assay, respectively, measured cell migration and invasion, at the concentration of $0.5 \mu \mathrm{M}$ nicotine for $24 \mathrm{~h}$ stimulate both the HCC (Huh7 and HepG2) cells; bar plot indicates the percentage speed of wound healing and invasion between the nicotine and control treatment group. Scale bar $50 \mu \mathrm{m}$. (c, d) Nicotine induces self-renewal, i.e., colony-forming and tumor-sphere properties of HCC cells. Scale bar $100 \mu \mathrm{m}$. (e) Protein expression by Western blotting in HCC cells treated with nicotine. Nicotine treatment $(0.5 \mu \mathrm{M})$ efficiently induced the expression of p-JAK2, EMT marker (induced vimentin), and self-renewal (CD133) markers. GAPDH served as the loading control. The bar represents the mean \pm SD values of three independent experiments; ${ }^{*} p<0.05$ versus controls (Student's $t$-test).

and 5(b)). Interestingly, tumor sphere and self-renewal markers, such as CD133 and SOX2, were significantly reduced at the protein level (Figure 5(c)) after treatment with DHCT compared to nicotine only treated group cells. FACS analysis also demonstrated the reduction in the generation of ALDH1+ cells in DHCT treated cells compared to the nicotine only treated group (Figure 5(d)). These results indicate DHCT potential in inhibiting the self-renewal phenotype of nicotine-stimulated HCC cells.

3.6. In Vivo Suppressive Effect of DHCT on Nicotine-Induced HepG2 Tumorigenesis. After establishing the DHCTs' antiHCC role on in vivo nude mice model. In vivo study, we evaluated the DHCT effects using a xenograft mice HepG2 tumor model. After the tumors were palpable in mice, the mice were randomly divided into three groups, control ( $n=5$; saline treatment only), nicotine $(n=5$; nicotinetreated), and DHCT+nicotine $(n=5$; DHCT+nicotinetreated) groups. The tumor volume was monitored once per week for another 5 weeks. As depicted in Figure 6(a), the nicotine-treated group mice are showing enhanced tumor growth as compared with that of the saline only control and DHCT+nicotine treatment group, suggesting the combination treatment of DHCT+nicotine leads to a significant reduction in tumor volume $\left({ }^{* *} p<0.01\right)$. Moreover, the overall survival time was higher in mice treated with the $\mathrm{DHCT}+$ nicotine combination compared with that in mice treated with nicotine and nontreated control groups (Figure 6(b)). Moreover, comparative real-time PCR analyses showed (Figure 6(c)) a reduced level of tumor aggressiveness Ki-67 expression markers, induced apoptotic Caspase- 3 and TUNEL-positive marker in the DHCT+nicotine combination group in comparison with saline and nicotine treatment. Furthermore, IHC analysis results of tissue sections in mice were consistent with the results of qRT-PCR. IHC result demonstrated that combination treatment of DHCT +nicotine suppresses the Ki-67 marker, i.e., tumor proliferation, oncogenicity, and tumorigenesis, and induced apoptosis was observed as compared with saline and nicotine treatment (Figure 6(d)) animal group.

\section{Discussion}

Nicotine, a major by-product of cigarette smoking, may be responsible for the initiation, progression, and therapy outcomes of various cancers [26]. It influences various biological processes by altering gene expression and inducing oxidative stress, DNA damage, apoptosis, cell proliferation, and angiogenesis $[26,27]$. It also contributes to carcinogenesis in various cancer types, affects cancer progression, and is associated with poor prognosis $[28,29]$. Nicotine binds to nAChRs and induces many downstream cancer-associated signaling pathways [29]. Epidemiological studies have reported that smoking tobacco significantly increases the initiation and progression of HCC [30].

Nicotine regulates many cancer-associated properties in most cancers and plays a vital role in inflammatory marker expression, which then regulates various other pathological conditions [8-11]. Many malignancies are associated with increased exposure to nicotine [29], notably HCC, which is a common type of liver cancer and is one of the most difficult to treat malignancies worldwide [31].

Our previous study highlighted smoking as an independent risk factor for HCC progression through $\alpha 7 \mathrm{nAChR}$ and JAK2 signaling. [16]. In this study, we investigated the mechanisms underlying nicotine's effects on HCC progression through $\alpha 7 \mathrm{nAChR}$ and JAK2 signaling and the reversal of those effects by DHCT, a novel kinase inhibitor.

In the present study, we demonstrated that nicotine treatment induced the HCC cells' proliferation, invasion, 


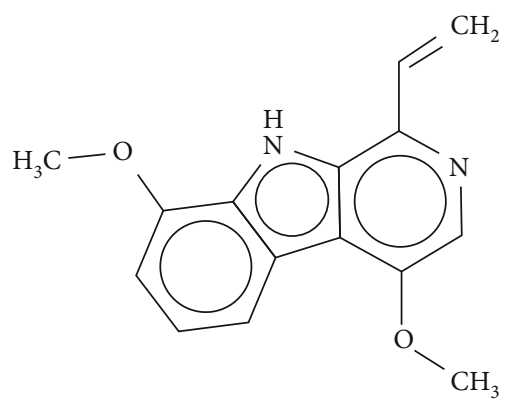

Dehydrocrenatidine (DHCT)

MW: 254.28

(a)
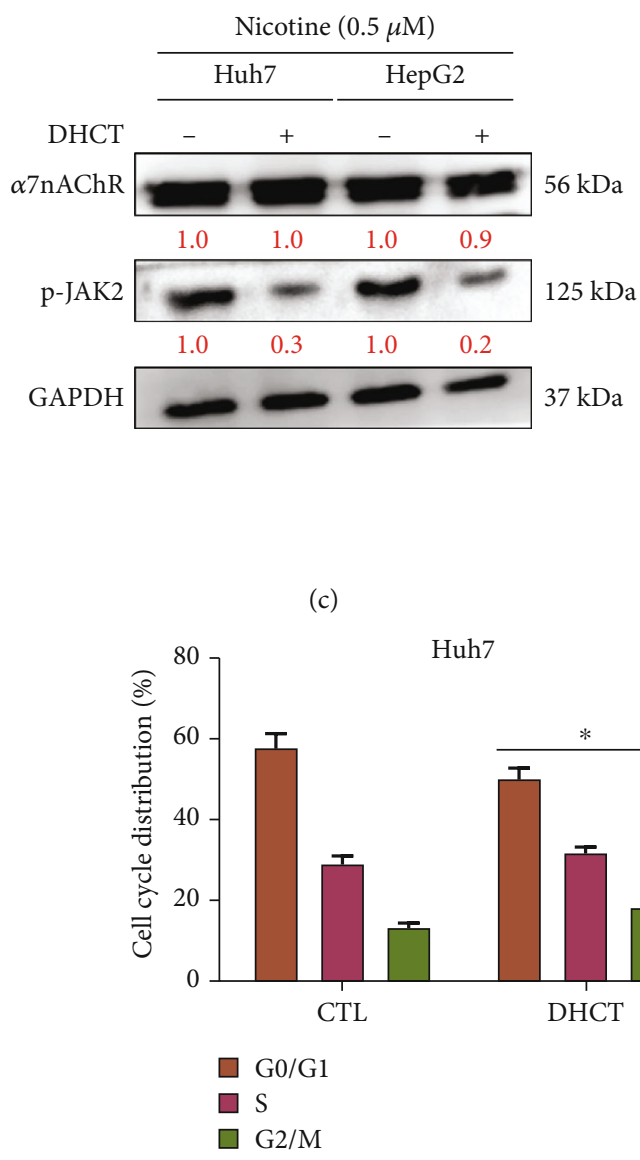

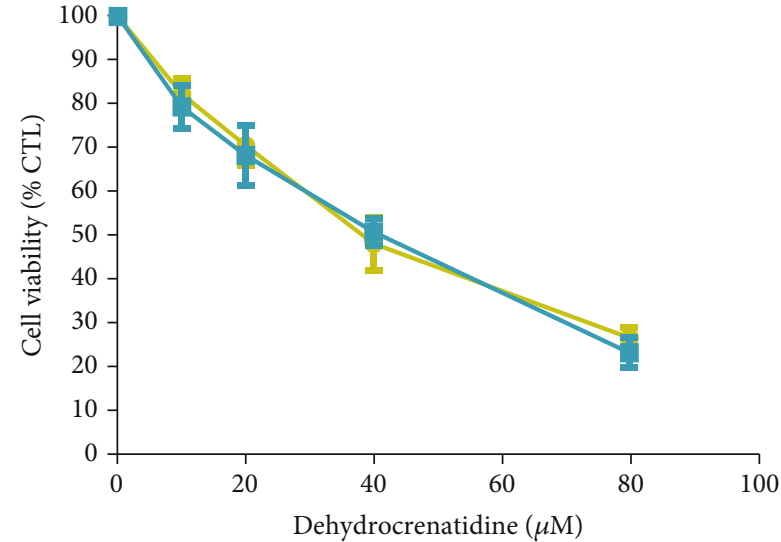

$$
\begin{aligned}
& - \text { HepG2, } \mathrm{IC}_{50}=36.8770 \\
& - \text { Huh7, } \mathrm{IC}_{50}=34.4410
\end{aligned}
$$

(b)

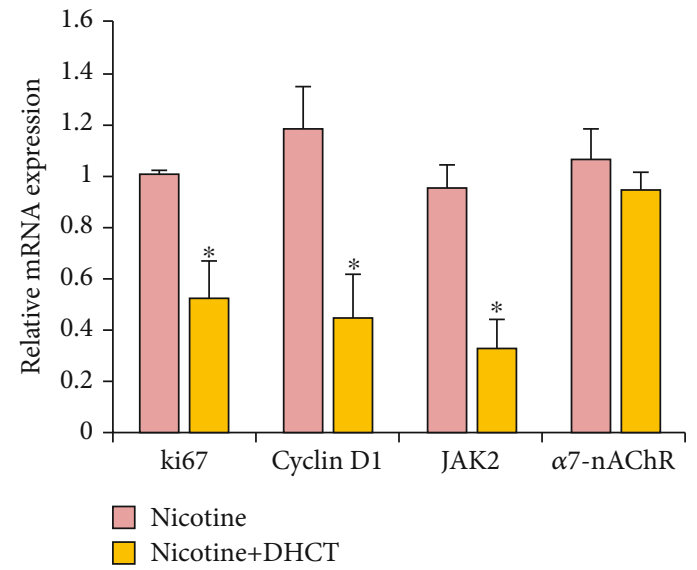

(d)

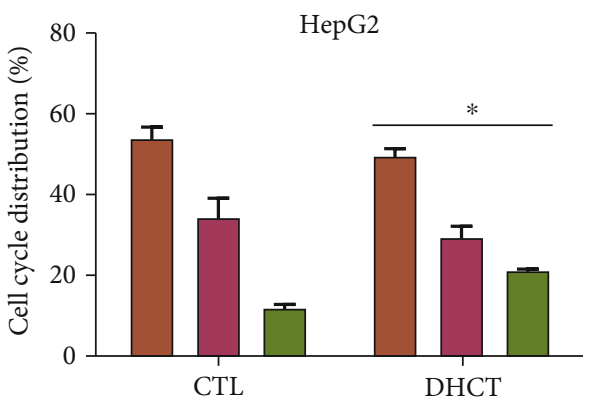

(e)

Figure 3: Dehydrocrenatidine (DHCT) modulates the nicotine treatment effect on HCC cells. (a) The image represents the chemical structure of DHCT. (b) Cell viability assay for $\mathrm{IC}_{50}$ evaluation of DHCT treatment on both Huh7 and HepG2 HCC cells were determined by CCK- 8 assay. (c) The relative reduction in protein expression of $\alpha 7 \mathrm{nAChR}$ and p-JAK2 by Western blotting, (d) qRTPCR analysis denoted the relative reduction at mRNA level of Ki67, Cyclin D1, JAK2, and $\alpha 7 n A C h R$ after DHCT treatment on nicotine stimulated cells. (e) DHCT-treated HCC cells (Huh7 and HepG2) cells were harvested; cell cycle was analyzed as the percentage of cells at each stage of the cell cycle after DNA staining with PI. Data from one of the representative experiments (from at least three independent assays); ${ }^{* *} p<0.01$ versus controls (Student's $t$-test). 

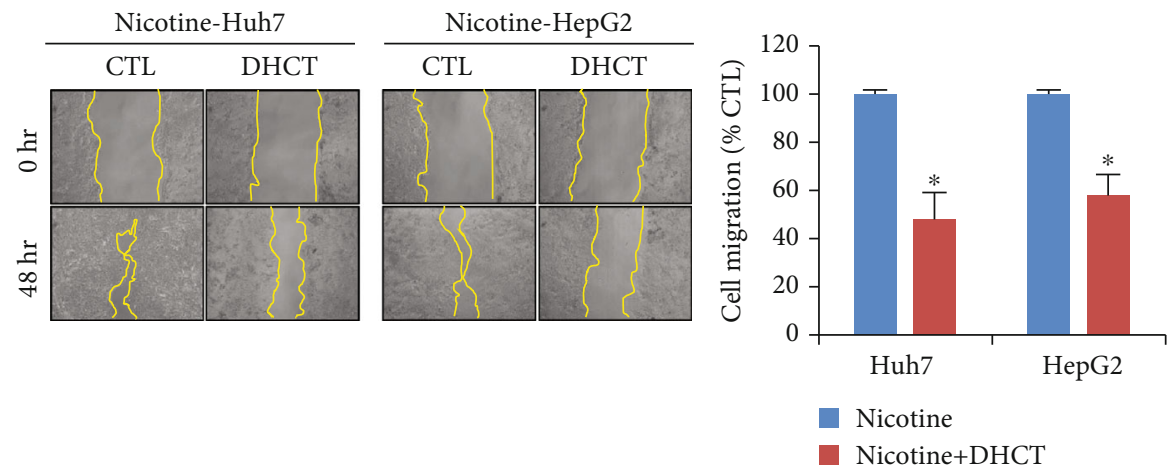

(a)
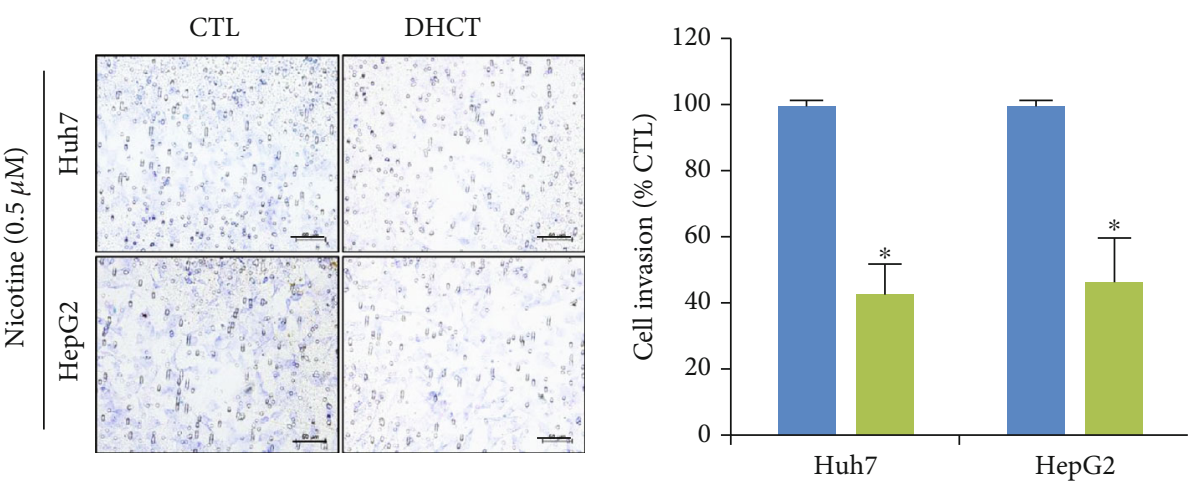

Nicotine

Nicotine+DHCT

(b)

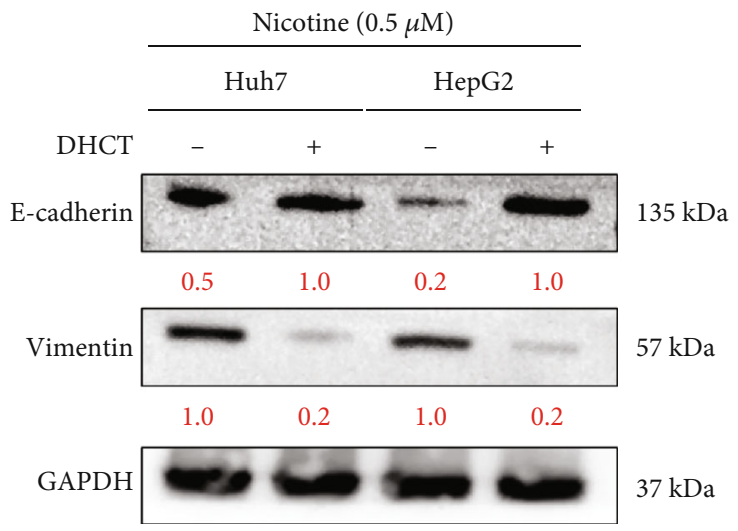

(c)

Figure 4: DHCT reversed nicotine induces cell migratory, invasive, and EMT properties in Huh7 and HepG2 HCC cells. (a, b) Wound healing and Transwell assay, respectively, measured cell migration and invasion, after the DHCT treatment on nicotine $(0.5 \mu \mathrm{M})$ stimulated HCC (Huh7 and HepG2) cells, bar plot adjacent indicates the percentage speed of wound healing and invasion between the DHCT + nicotine and nicotine treatment group. Scale bar $50 \mu \mathrm{m}$. (c) Western blot analysis of protein expression on HCC cells treated with DHCT efficiently reduced the expression of EMT process; induced E-cadherin, and reduced vimentin expression observed. GAPDH served as the loading control. Data from one of a representative experiment, (from at least three independent assays); ${ }^{*} p<0.05$ versus controls (Student's $t$-test).

and self-renewal abilities (Figures 1 and 2) by stimulating and inducing $\alpha 7 \mathrm{nACh}$, JAK2, Ki67, and cyclin D1 expression (Figure 1). Ki67 and cyclin D1 expression indicate the proliferative abilities of cancer cells [32]. Moreover, nicotine exposure significantly modulates the EMT [33], which is associated with invasion, metastasis, and self-renewal (CSCs)
[34] of HCC cancer cells (Figure 2). This agrees with recent studies reporting that nicotine exposure stimulates $\alpha 7 \mathrm{nAChR}$ expression in cancer cells [35]. de Jonge et al. [18] and Krafft et al. [36] have demonstrated that the expressions of $\alpha 7 \mathrm{nAChR}$ and JAK2 are strongly correlated. Notably, $\alpha 7 \mathrm{nAChR}$ and JAK2 expression was associated with poor 


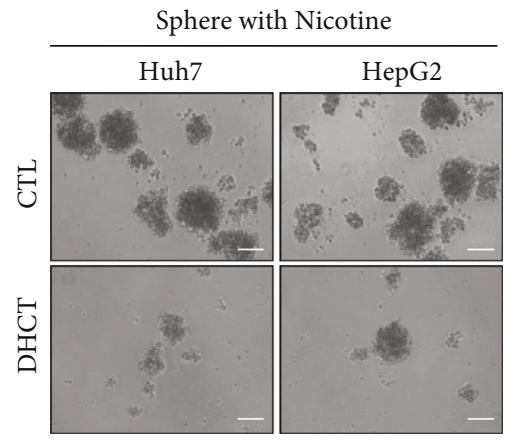

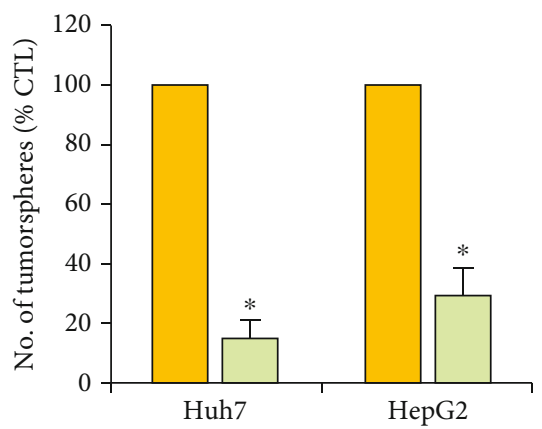

Nicotine

Nicotine+DHCT

(a)

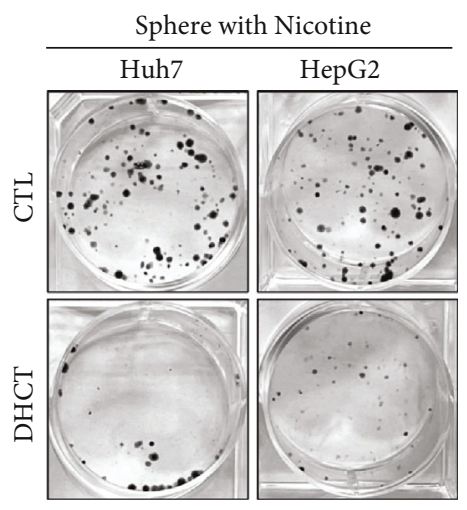

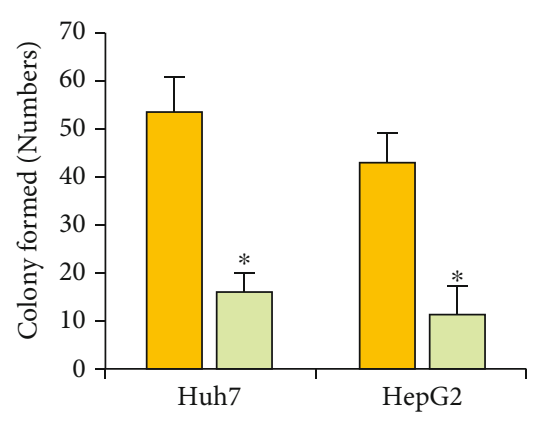

Nicotine

Nicotine+DHCT

(b)

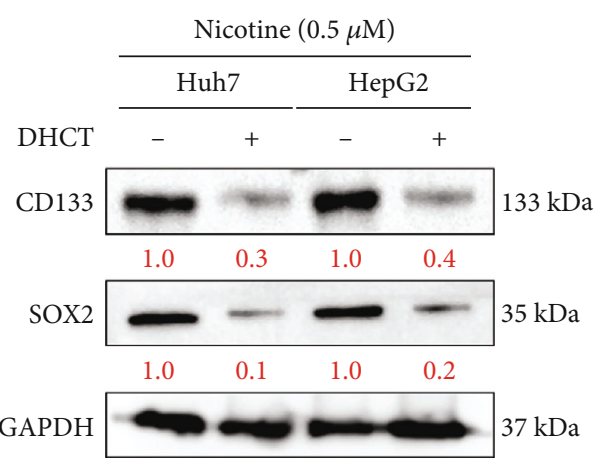

(c)

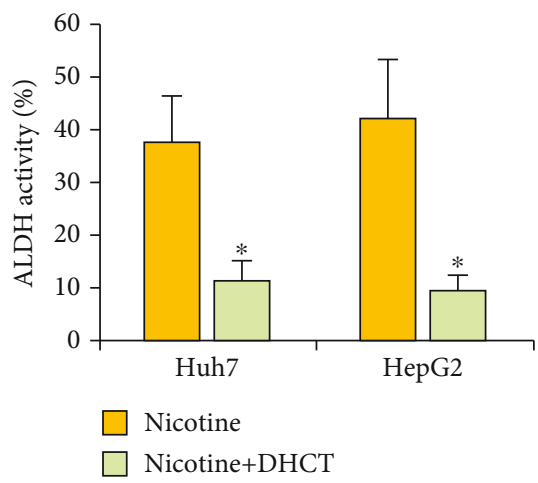

(d)

Figure 5: DHCT reversed nicotine effects on tumor sphere and colony-forming abilities of Huh7 and HepG2 HCC cells. (a, b) Representative image of DHCT treatment on the self-renewal, i.e., tumor-sphere and colony-forming properties of HCC cells. Scale bar $100 \mu \mathrm{m}$. (c) Detection of the expression of self-renewal (CD133 and SOX2) markers. GAPDH served as the loading control. (d) Representative bar graph of fluorescence-activated cell sorting (FACS) showing reduction of $\mathrm{ALDH}^{+}$cancer stem-like cell population of DHCT-treated HCC cell line compared to nontreated control; ${ }^{*} p<0.05 ;{ }^{* *} p<0.051$ versus controls (Student's $t$-test).

overall survival in patients with HCC $[16,37]$. Thus, the $\alpha 7 \mathrm{nAChR}$ gene might play a key role in HCC through JAK2 signaling. Furthermore, DHCT significantly inhibited the in vitro growth and proliferation of HCC cells and sensitized them to nicotine treatment. DHCT treatment reduced the expression of $\alpha 7 \mathrm{nAChR}$, JAK2, and markers of prolifera- tion and cell cycle arrest (Figure 3). Regarding in vitro mobility, migration and invasion are associated with the metastatic potential of HCC cells [38]. Our data confirmed (Figure 4) that DHCT effectively inhibited the effects of $\alpha 7 \mathrm{nAChR}$ and JAK2 in nicotine-treated HCC cells, which subsequently reduced their migration/invasion abilities by disturbing the 


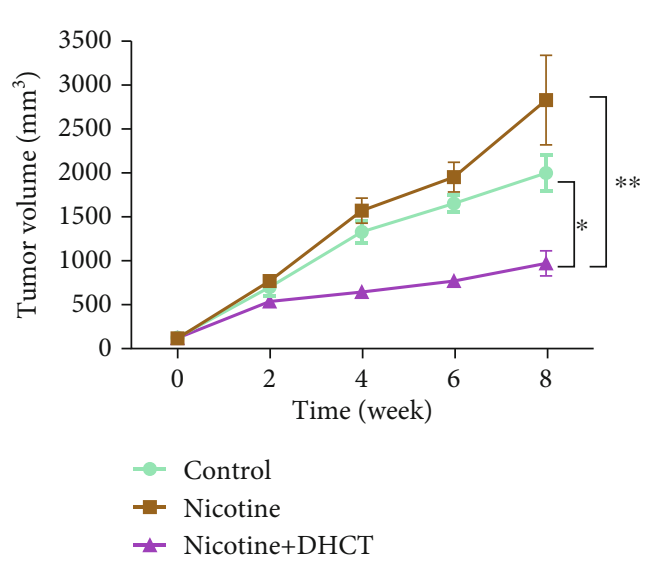

(a)

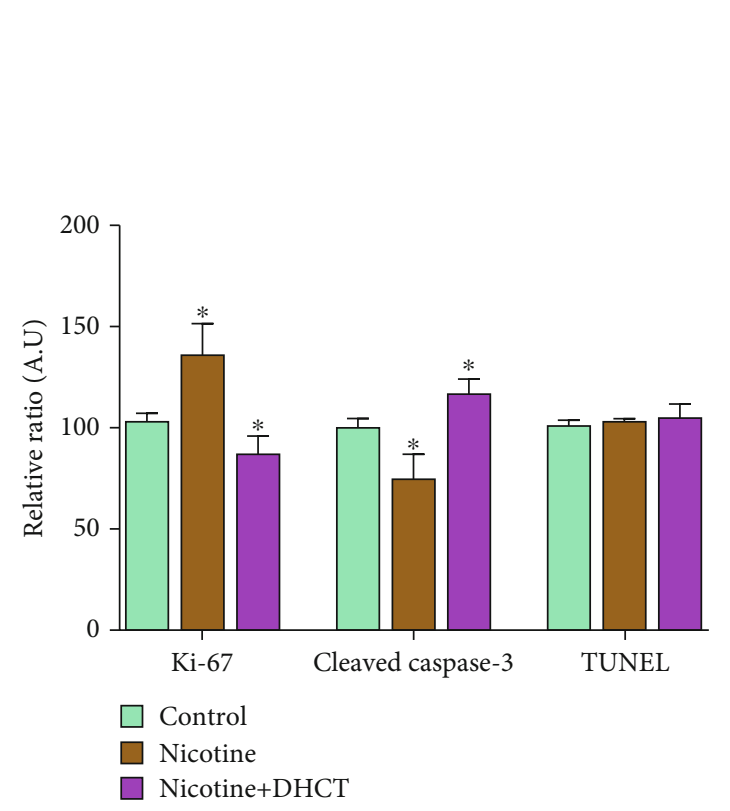

(c)

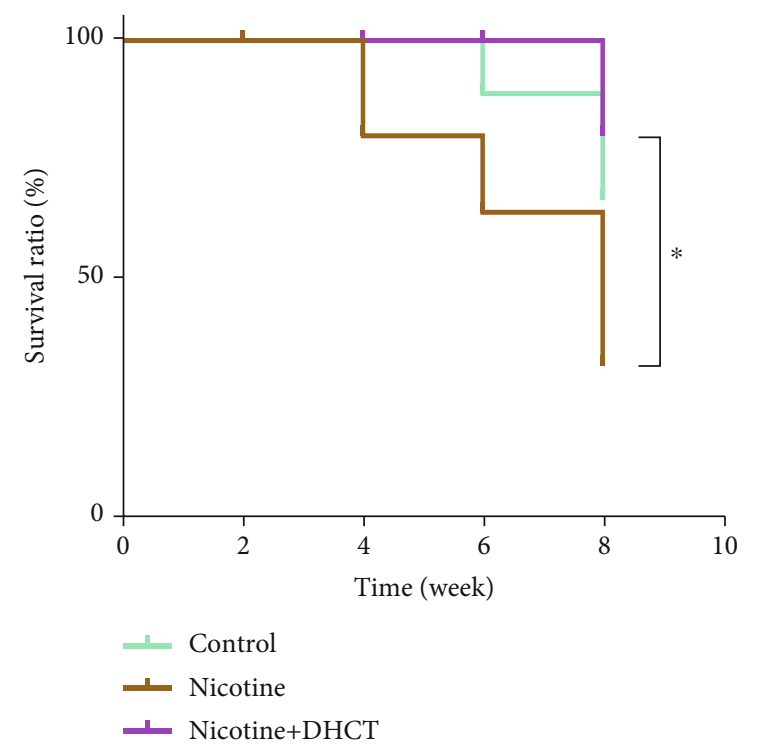

(b)

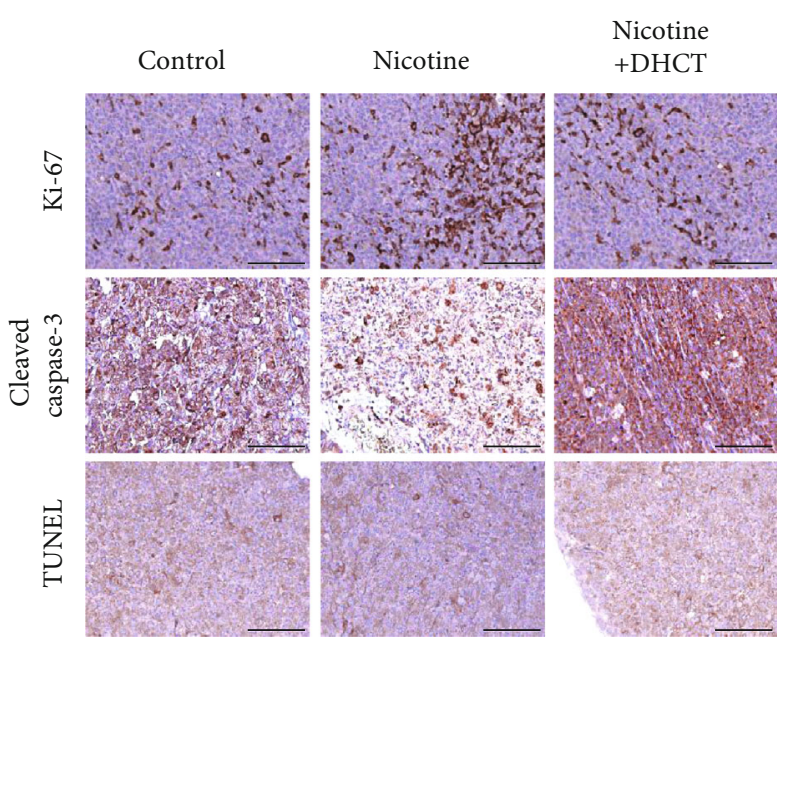

(d)

FIgURE 6: The combination of DHCT+nicotine inhibits tumor growth in HCC mice models. (a) The tumor volume over the time curve was seen in combination treatment, as compared to the nicotine and saline control individual treatment groups. (b) Kaplan-Meier survival curve showed the best overall survival ratio in DHCT+nicotine combination, compared to nicotine only and saline control treatment groups. (c) Comparative real-time PCR analyses showed a significantly reduced Ki-67 expression and induced apoptosis (cl-Caspase-3 and TUNELpositive cells) mRNA level in combination groups as compared with nicotine and saline counterparts. (d) Representative hematoxylin and eosin $(\mathrm{H} \& \mathrm{E})$ stained images are shown, and IHC detected the expression of Ki-67 and cl-Caspase-3, and TUNEL apoptotic cells in the tumors from mice were detected. ${ }^{*} P<0.05$, and ${ }^{* *} P<0.01$.

expression of EMT biomarkers (E-cadherin and vimentin) and a transcription factor (Slug).

Often, patients with HCC have chemoresistance, which is enhanced by nicotine exposure (Figure 2). The expression of CSCs is connected to drug resistance [34], which is a major challenge in HCC therapy [39]. CSCs contribute to tumorigenicity, self-renewal abilities, and chemoresistance [40, 41]. Notably, DHCT treatment of HCC cells resulted in the reduced expression of $\alpha 7 \mathrm{nAChR}$, JAK2, and cancer stemness markers (CD133, KLF4, and SOX2) as well as decreased ALDH1 activity (Figure 5), thereby suppressing the colony-forming and tumor sphere generation abilities of HCC cells.

To further confirm the effect of DHCT in modulating and sensitizing the nicotine effect in vivo. HepG2 cells were subcutaneously injected into the xenograft mice models. 


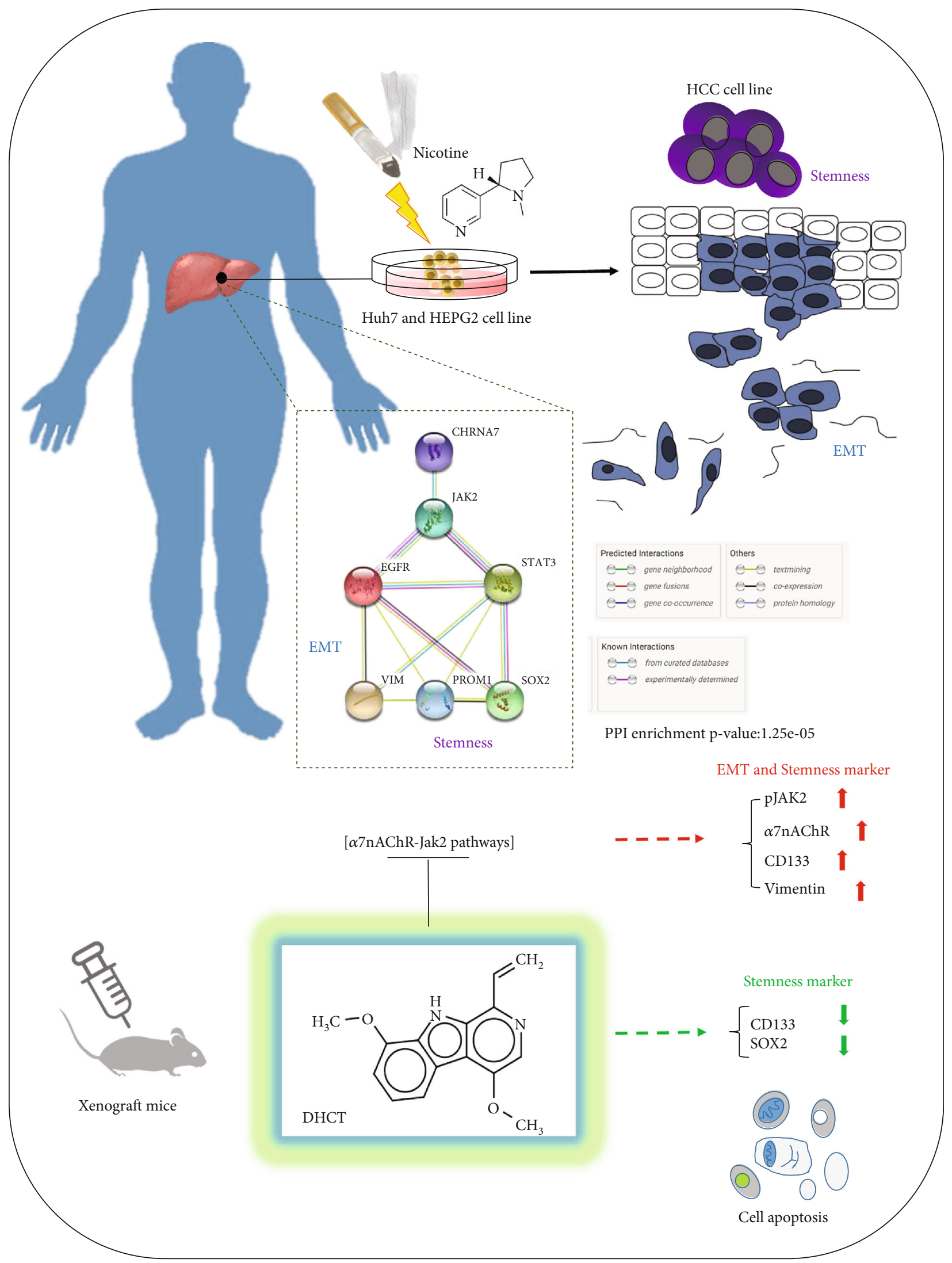

FIGURE 7: A pictorial abstract showing the natural compound dehydrocrenatidine regulating the a7nAChR-Jak2 signaling pathways attenuates nicotine-induced stemness and epithelial-mesenchymal transition in hepatocellular carcinoma and a series of experimental designs. 
Nicotine alone or in combination with DHCT+nicotine and saline control was administered. Reduction in tumor burden, reduced expression of $\mathrm{Ki}-67$, and induced tumor apoptosis were observed in the DHCT+nicotine treatment group, which results in the reduction/neutralizing effect of nicotine on tumor-initiating abilities in xenograft models (Figure 6).

\section{Conclusion}

In conclusion, as shown in the pictorial abstract of Figure 7 , it demonstrated that the natural compound dehydrocrenatidine attenuates nicotine-induced HCC cell stemness and EMT potential through a7nAChR-Jak2 signaling disruption and a series of experimental designs. Our results revealed that nicotine exposure-induced tumorigenicity in HCC cells by inducing the expression of $\alpha 7 \mathrm{nAChR}$ and JAK2, which was effectively suppressed by DHCT treatment. This led to a reduction of CSC genes and antitumor efficacy in HCC cells. Thus, DHCT treatment could overcome/reverse the nicotine-induced progression of HCC cells, which may serve as a useful therapeutic strategy in patients with HCC.

\section{Data Availability}

The datasets used and analyzed in the current study are publicly accessible as indicated in the manuscript.

\section{Additional Points}

Highlights. (i) Natural compound dehydrocrenatidine attenuates nicotine-induced stemness in hepatocellular carcinoma. (ii) Natural compound dehydrocrenatidine attenuates nicotine-induced epithelial-mesenchymal transition in hepatocellular carcinoma. (iii) Natural compound dehydrocrenatidine regulating a7nAChR-Jak2 signaling pathways in hepatocellular carcinoma.

\section{Conflicts of Interest}

We confirm that we have no known conflicts of interest associated with this publication, and no significant financial support for this work could have influenced its outcome.

\section{Authors' Contributions}

Ching-Li $\mathrm{Li}$ is responsible for the study conception and experimental design; Chien-Che Wang, Hsin-Te Pai, StanLey $\mathrm{Tu}$, and Pin-Yuan Hou performed the experiments; Chien-Yu Huang is responsible for the data collation and analysis; Ming-Te Huang and Yu-Jia Chang for the manuscript writing; Ming-Te Huang and Yu-Jia Chang for the provided reagents, materials, and experimental infrastructure; and all authors read and approved the final submitted version of the manuscript.

\section{Acknowledgments}

The authors thank all research assistants of the Translational Research Laboratory and Core Facility Center, Taipei Medi- cal University-Shuang Ho Hospital, for their assistance with the flow cytometry and molecular and cell-based assays. The author also thanks the Gene Targeting Core Lab at Taipei Medical University in Taiwan for providing technical support. The authors would like to acknowledge Taipei Medical University for the technical support of professional English proofreading and editing services.

\section{Supplementary Materials}

Supplementary Table S1: Western blot antibodies sheet. Supplementary Table S2: primer sequence in this study. (Supplementary Materials)

\section{References}

[1] S. E. Jackson, J. Brown, L. Shahab, A. Steptoe, and D. Fancourt, "Covid-19, smoking and inequalities: a study of 53002 adults in the uk," tobaccocontrol, vol. 30, no. e2, pp. e111-e121, 2020.

[2] A.-R. El-Zayadi, "Heavy smoking and liver," World Journal of Gastroenterology, vol. 12, no. 38, pp. 6098-6101, 2006.

[3] S. P. Saha, D. K. Bhalla, T. F. Whayne Jr., and C. Gairola, "Cigarette smoke and adverse health effects: an overview of research trends and future needs," The International journal of angiology: official publication of the International College of Angiology, Inc, vol. 16, no. 3, pp. 77-83, 2007.

[4] A. Mishra, P. Chaturvedi, S. Datta, S. Sinukumar, P. Joshi, and A. Garg, "Harmful effects of nicotine," Indian J Med Paediatr Oncol, vol. 36, no. 1, pp. 24-31, 2015.

[5] J. Wu, "Understanding of nicotinic acetylcholine receptors," Acta Pharmacologica Sinica, vol. 30, no. 6, pp. 653-655, 2009.

[6] K. Hajiasgharzadeh, M. H. Somi, B. Mansoori et al., "Alpha7 nicotinic acetylcholine receptor mediates nicotine-induced apoptosis and cell cycle arrest of hepatocellular carcinoma hepg2 cells," Adv Pharm Bull, vol. 10, no. 1, pp. 65-71, 2020.

[7] C. Ren, Y.-L. Tong, J.-C. Li, Z.-Q. Lu, and Y.-M. Yao, "The protective effect of alpha 7 nicotinic acetylcholine receptor activation on critical illness and its mechanism," International Journal of Biological Sciences, vol. 13, no. 1, pp. 46-56, 2017.

[8] H. M. Schuller, "Regulatory role of the ?7nAChR in cancer," Current Drug Targets, vol. 13, no. 5, pp. 680-687, 2012.

[9] C. C. Tu, C. Y. Huang, W. L. Cheng, C. S. Hung, Y. J. Chang, and P. L. Wei, "Silencing a7-nachr levels increases the sensitivity of gastric cancer cells to ixabepilone treatment," Tumour Biology, vol. 37, no. 7, pp. 9493-9501, 2016.

[10] L. Zhang, L. Wu, H. Huan et al., "Sympathetic and parasympathetic innervation in hepatocellular carcinoma," Neoplasma, vol. 64, no. 6, pp. 840-846, 2017.

[11] H. P. S. Wong, L. Yu, E. K. Y. Lam, E. K. K. Tai, W. K. K. Wu, and C. H. Cho, "Nicotine promotes cell proliferation via $\alpha 7$ nicotinic acetylcholine receptor and catecholaminesynthesizing enzymes-mediated pathway in human colon adenocarcinoma ht-29 cells," Toxicology and Applied Pharmacology, vol. 221, no. 3, pp. 261-267, 2007.

[12] T. Sanner and T. K. Grimsrud, "Nicotine: carcinogenicity and effects on response to cancer treatment-a review," Frontiers in Oncology, vol. 5, p. 196, 2015.

[13] P. Rawla, T. Sunkara, P. Muralidharan, and J. P. Raj, "Update in global trends and aetiology of hepatocellular carcinoma," Contemporary Oncology, vol. 22, no. 3, pp. 141-150, 2018. 
[14] R. Kumari, M. K. Sahu, A. Tripathy, K. Uthansingh, and M. Behera, "Hepatocellular carcinoma treatment: hurdles, advances and prospects," Hepat Oncol, vol. 5, no. 2, p. HEP08-HEP08, 2018.

[15] J. L. Petrick, P. T. Campbell, J. Koshiol et al., “Tobacco, alcohol use and risk of hepatocellular carcinoma and intrahepatic cholangiocarcinoma: the liver cancer pooling project," British Journal of Cancer, vol. 118, no. 7, pp. 1005-1012, 2018.

[16] C. L. Li, Y. K. Lin, H. A. Chen, C. Y. Huang, M. T. Huang, and Y. J. Chang, "Smoking as an independent risk factor for hepatocellular carcinoma due to the $\alpha 7$-nachr modulating the jak2/ stat3 signaling axis," Journal of Clinical Medicine, vol. 8, no. 9, p. 1391, 2019.

[17] K. J. Tracey, "Physiology and immunology of the cholinergic antiinflammatory pathway," The Journal of Clinical Investigation, vol. 117, no. 2, pp. 289-296, 2007.

[18] W. J. de Jonge, E. P. van der Zanden, F. O. The et al., "Stimulation of the vagus nerve attenuates macrophage activation by activating the jak2-stat3 signaling pathway," Nature Immunology, vol. 6, no. 8, pp. 844-851, 2005.

[19] W. Jin, "Role of jak/stat3 signaling in the regulation of metastasis, the transition of cancer stem cells, and chemoresistance of cancer by epithelial-mesenchymal transition," Cell, vol. 9, no. 1, p. 217, 2020.

[20] H. Yu, D. Pardoll, and R. Jove, "Stats in cancer inflammation and immunity: a leading role for stat3," Nature Reviews. Cancer, vol. 9, no. 11, pp. 798-809, 2009.

[21] H. Lee, A. J. Jeong, and S.-K. Ye, "Highlighted stat3 as a potential drug target for cancer therapy," BMB Reports, vol. 52, no. 7, pp. 415-423, 2019.

[22] J. Zhang, N. Zhu, Y. du et al., "Dehydrocrenatidine is a novel janus kinase inhibitor," Molecular Pharmacology, vol. 87, no. 4, pp. 572-581, 2015.

[23] D. R. Rhodes, J. Yu, K. Shanker et al., "ONCOMINE: A Cancer Microarray Database and Integrated Data-Mining Platform," Neoplasia (New York, N.Y.), vol. 6, no. 1, pp. 1-6, 2004.

[24] N. A. Franken, H. M. Rodermond, J. Stap, J. Haveman, and C. van Bree, "Clonogenic assay of cells_in vitro_," Nature Protocols, vol. 1, no. 5, pp. 2315-2319, 2006.

[25] L. P. Schwab, D. L. Peacock, D. Majumdar et al., "Hypoxiainducible factor $1 \alpha$ promotes primary tumor growth and tumor-initiating cell activity in breast cancer," Breast cancer research: BCR, vol. 14, no. 1, p. R6, 2012.

[26] R. Zeidler, K. Albermann, and S. Lang, "Nicotine and apoptosis," Apoptosis, vol. 12, no. 11, pp. 1927-1943, 2007.

[27] J. Lee and J. P. Cooke, "Nicotine and pathological angiogenesis," Life Sciences, vol. 91, no. 21-22, pp. 1058-1064, 2012.

[28] A. W. Bergen and N. Caporaso, "Cigarette smoking," Journal of the National Cancer Institute, vol. 91, no. 16, pp. 13651375, 1999.

[29] S. A. Grando, "Connections of nicotine to cancer," Nature Reviews Cancer, vol. 14, no. 6, pp. 419-429, 2014.

[30] X.-F. Zhang, T. Wei, X.-M. Liu, C. Liu, and Y. Lv, "Impact of cigarette smoking on outcome of hepatocellular carcinoma after surgery in patients with hepatitis b," PLoS One, vol. 9, no. 1, article e85077, 2014.

[31] K. Hajiasgharzadeh, M. H. Somi, D. Shanehbandi, A. Mokhtarzadeh, and B. Baradaran, "Small interfering rnamediated gene suppression as a therapeutic intervention in hepatocellular carcinoma," Journal of Cellular Physiology, vol. 234, no. 4, pp. 3263-3276, 2019.
[32] M. Joo, Y. K. Kang, M. R. Kim, H. K. Lee, and J. J. Jang, "Cyclin d1 overexpression in hepatocellular carcinoma," Liver, vol. 21, no. 2, pp. 89-95, 2001.

[33] A. Suetsugu, M. Nagaki, H. Aoki, T. Motohashi, T. Kunisada, and H. Moriwaki, "Characterization of $\mathrm{cd} 133^{+}$hepatocellular carcinoma cells as cancer stem/progenitor cells," Biochemical and Biophysical Research Communications, vol. 351, no. 4, pp. 820-824, 2006.

[34] V. S. Donnenberg and A. D. Donnenberg, "Multiple drug resistance in cancer revisited: the cancer stem cell hypothesis," Journal of Clinical Pharmacology, vol. 45, no. 8, pp. 872-877, 2005.

[35] P. Russo and A. Taly, "A7-nicotinic acetylcholine receptors: an old actor for new different roles," Current Drug Targets, vol. 13, no. 5, pp. 574-578, 2012.

[36] P. R. Krafft, D. McBride, W. B. Rolland, T. Lekic, J. J. Flores, and J. H. Zhang, " $\alpha 7$ Nicotinic Acetylcholine Receptor Stimulation Attenuates Neuroinflammation through JAK2-STAT3 Activation in Murine Models of Intracerebral Hemorrhage," BioMed Research International, vol. 2017, 13 pages, 2017.

[37] F. SONOHARA, S. NOMOTO, Y. INOKAWA et al., "High expression of janus kinase 2 in background normal liver tissue of resected hepatocellular carcinoma is associated with worse prognosis," Oncology Reports, vol. 33, no. 2, pp. 767-773, 2015.

[38] Y. Li, B. Tian, J. Yang et al., "Stepwise metastatic human hepatocellular carcinoma cell model system with multiple metastatic potentials established through consecutive in vivo selection and studies on metastatic characteristics," Journal of Cancer Research and Clinical Oncology, vol. 130, no. 8, pp. 460-468, 2004.

[39] H. Huynh, R. Ong, K. Y. Goh et al., "Sorafenib/mek inhibitor combination inhibits tumor growth and the wnt/ $\beta$-catenin pathway in xenograft models of hepatocellular carcinoma," International Journal of Oncology, vol. 54, no. 3, pp. 11231133, 2019.

[40] J. Neuzil, M. Stantic, R. Zobalova et al., "Tumour-initiating cells vs. cancer 'stem' cells and cd133: what's in the name?," Biochemical and Biophysical Research Communications, vol. 355, no. 4, pp. 855-859, 2007.

[41] A. Scopelliti, P. Cammareri, V. Catalano, V. Saladino, M. Todaro, and G. Stassi, "Therapeutic implications of cancer initiating cells," Expert Opinion on Biological Therapy, vol. 9, no. 8, pp. 1005-1016, 2009. 